\title{
Seismic imaging of complex structures with the CO-CDS stack method
}

\author{
Amin Shahbazi (1), Mehrdad Soleimani (2), Vinesh Thiruchelvam (1), and Ehsan Sharifi (3)
}

(1) Asia Pacific University,Malaysia (amin.shahbazi@hotmail.com), (2) Shahrood University of Technology (mehrdad_soleimani2005@yahoo.co.nz), (3) University of Vienna (ehsansharifi83@gmail.com)

\begin{abstract}
Various seismic imaging methods are introduced to resolve some of the possible ambiguities of seismic interpretation in complex structures. Reducing dependency of imaging techniques on velocity or using diffraction energy for imaging more structural details are the main topics of the imaging research. In this study, we try to improve the seismic image quality in semi-complex structures by combining the common reflection surface (CRS) method with a diffraction based scheme in the common-offset domain. Previously introduced partial CRS and common offset CRS methods exhibited reliable performance in imaging complex media. Here, we were looking for stable and efficient solutions, preserving advantages of the previous methods. Herewith, the proposed operator fits better to diffractions than to reflections. Therefore, we call it the commonoffset common diffraction surface stack (CO CDS). In a previous study, improvement of the quality of seismic image by the CRS method was achieved by combination of the CDS method with the partial CRS. This resulted in the introduction of the partial CDS. Initially, in this study, the common-offset CRS traveltime equation was modified to the common-offset CDS. The hypothetical shot reflector experiment in the CRS method was changed to shot diffraction point experiment. In the introduced operator, two wavefront curvatures, observed at receivers positions, are set equal in order to satisfy the diffraction condition. In the proposed method, we search for accurate attribute sets for each considered offset individually, and then form a new operator by four coherent attributes. Application of the common- offset CDS method on synthetic and field data shows more details of the geological structures with higher quality, while preserving continuity of reflection events. The proposed method is, however, more expensive than the partial and common offset CRS for large dataset.
\end{abstract}

